\title{
Les eaux résiduaires dans l'industrie laitière
}

\author{
par \\ J.L. BURGAUD \\ Docteur en Chimie
}

Le problème du rejet des eaux usées industrielles prend chaque jour davantage d'importance et cette évolution est particulièrement nette dans les usines laitières qui doivent toutes envisager d'avoir à épurer, dans un avenir plus ou moins proche, leurs eaux polluées.

Cet état de fait est dû à deux causes principales :

- Le renforcement généralisé de la législation concernant les eaux résiduaires.

- La transformation des usines traitant le lait et qui deviennent de plus en plus importantes. Il existe maintenant des usines de très grandes tailles qui utilisent de grandes quantités d'eau et qui rejettent des masses de polluants qui sont sans commune mesure avec celles provenant des petites installations d'autrefois. Il en résulte que les tolérances qui étaient alors admissibles ou les méthodes qui étaient utilisées ne sont plus actuellement possibles.

Nous allons essayer, dans cet article, de définir les principaux aspects de ce problème et de mettre en évidence les moyens à mettre en œuvre pour le résoudre.

Nous envisagerons successivement :

- L'état actuel de la législation.

- Les polluants des eaux de laiterie.

- L'origine des eaux résiduaires de laiterie.

- Les techniques applicables à leur épuration.

\section{I. - LA LEGISLATION}

La législation actuellement en vigueur fait intervenir deux notions particulières que sont la $\mathrm{DBO}$ et la $\mathrm{DCO}$, et que nous allons définir rapidement :

- La DBO ou demande biochimique en oxygène s'exprime en $\mathrm{mg} / \mathrm{l}$ d'oxygène. On mesure pour la définir l'abaissement en un temps donné, de la concentration en oxygène dissous, d'une solution 
contenant une certaine quantité de l'échantillon à analyser. Le plus souvent la mesure se fait sur 5 jours et on parle de DBO 5 .

La DBO est une mesure de l'activité biologique qui existe dans les eaux polluées. Celles-ci contiennent des micro-organismes qui, en présence d'oxygène, "consomment " certains produits organiques contenus dans l'eau. Cette activité biologique entraîne une disparition de ces produits organiques, qui sont oxydés ou qui servent à la synthèse de matière vivante, et une disparition d'oxygène, celui-ci étant nécessaire à l'activité des micro-organismes.

Ce processus se produit dans les rivières et est à la base de son auto-épuration. Il a pour conséquence de diminuer la teneur en oxygène dissous et peut de ce fait nuire à la vie aquatique.

La DBO doit le plus souvent se mesurer sur des solutions diluées. En effet, la quantité d'oxygène disponible est au plus la concentration de saturation de ce gaz dans l'eau, soit environ $10 \mathrm{mg} / \mathrm{l}$. $\mathrm{Si}$ les eaux à analyser ont une $\mathrm{DBO}$ supérieure à cette valeur, il est alors nécessaire de les diluer dans une solution ayant une DBO nulle ou très faible.

On voit que la DBO est une mesure globale qui ne fait pas intervenir la nature chimique des polluants. Certains produits qui ne sont pas biodégradables, donnent une valeur nulle pour la DBO. Il apparaît donc qu'il peut exister une pollution même en l'absence de DBO.

- La DCO est la demande chimique en oxygène. Elle s'exprime en mg/l d'oxygène. La DCO est la quantité d'oxygène fournie par un oxydant chimique nécessaire pour oxyder l'échantillon à analyser.

La valeur de la DCO va dépendre des conditions opératoires et de l'oxydant chimique utilisé. Plus celui-ci sera puissant, plus pour une eau donnée, la DCO sera élevée.

En France on applique maintenant la méthode de bichromate de potassium en milieu sulfurique au $1 / 2$, après ébullition de $2 \mathrm{~h}$. Il s'agit de conditions très puissantes d'oxydation et qui donnent pour la DCO les résultats les plus élevés.

La DCO comme la DBO, est une méthode de mesure globale qui ne donne aucun renseignement sur la nature des produits qui sont à l'origine de cette DCO.

Il n'y a, a priori, aucune proportionnalité entre les valeurs de la DBO et celles de la DCO. On peut cependant noter que plus le rapport $\frac{\mathrm{DBO}}{\mathrm{DCO}}$ est élevé, plus les polluants présents dans l'eau examinée seront facilement biodégradables, c'est-à-dire éliminables par des procédés biologiques d'épuration. 


\subsection{Normes de rejet}

L'instruction ministérielle du 6 juin 1953, toujours en vigueur, définit des normes pour le rejet des effluents industriels dans le milieu naturel. Les valeurs limites sont les suivantes :

- $\mathrm{pH}$ compris entre 5,5 et 8,5 .

- Température $\leqslant 30^{\circ} \mathrm{C}$.

- Composés cycliques hydroxylés ou leurs dérivés halogènes : 0 .

DBO 5 teneur maximum comprise entre 40 et $200 \mathrm{mg} / 1$ selon le rapport de débit entre l'effluent et le milieu récepteur. La valeur la plus basse $(40 \mathrm{mg} / 1)$ s'applique lorsque le débit des eaux usées dépasse le $1 / 150$ du débit de la rivière à l'étiage (si le rejet est continu sur $24 \mathrm{~h}$ ) ou le 1/360 du débit de la rivière lorsque le rejet n'a lieu que $10 \mathrm{~h}$ par jour. Dans la très grande majorité des cas, c'est la valeur à considérer.

Insolubles : teneur maximum comprise entre 30 et $100 \mathrm{mg} / \mathrm{l}$ selon le rapport des débits entre l'effluent et le milieu récepteur. Les rapports à considérer sont les mêmes que pour la DBO 5 .

Azote total organique compris entre 10 et $60 \mathrm{mg} / 1$ : la teneur limite à considérer dépendant du même paramètre que pour la DBO 5 et les insolubles.

Enfin une prescription supplémentaire interdit le rejet de toutes substances susceptibles de détruire la faune aquatique. Il est très souvent fait référence à cette clause malgré la difficulté d'apporter des preuves formelles surtout lorsqu'il s'agit d'une pollution accidentelle.

De très nombreux organismes officiels sont susceptibles d'entrer en contact avec un industriel dont les eaux rejetées sont suspectées : citons, par exemple le Service des Etablissements Classés, les Ponts et Chaussés, les Services de Santé ou de la Construction et la Direction Départementale de l'Agriculture.

Les sanctions prévues sont variées. Elles peuvent aller jusqu'à la fermeture de l'établissement incriminé. En fait, on a dans le passé fait preuve d'une grande souplesse dans l'application des textes et les difficultés principales que rencontraient les usines laitières, du fait de leurs rejets, étaient dues aux plaintes des pêcheurs. Elles se traduisaient dans beaucoup de cas par une simple amende.

\subsection{Organismes de bassin.}

Une loi de 1964 a découpé la France en bassins hydrologiques et créé pour chacun d'eux un Comité de bassin, organe consultatif composé de représentants de l'administration, des collectivités locales et des usagers.

A chaque Comité de bassin est associée une Agence Financière de Bassin qui est un établissement public administratif doté de la 
personnalité civile et de l'autonomie financière. Il est chargé de faciliter les diverses actions d'intérêt commun au bassin.

Les Agences Financières de Bassins doivent disposer de ressources qui lui permettront de faire face à leurs obligations. Pour ce faire elles sont habilitées à percevoir des taxes. Actuellement deux sont prévues :

- Taxe sur les prélèvements d'eau dans le milieu naturel (si l'eau utilisée provient d'un réseau public de distribution leur montant est compris dans le prix de l'eau).

- Taxe sur les rejets. Celle-ci ne donne pas un droit de pollution. Son paiement ne supprime donc aucune des difficultés qui peuvent résulter de la non observation des normes de rejet.

Les taxes sont dues directement à l'Agence, si le rejet a lieu dans le milieu naturel. Si les eaux usées sont envoyées dans un réseau communal ou intercommunal d'assainissement, c'est le propriétaire de ce réseau qui recouvrera auprès des utilisateurs les taxes qu'il devra payer à l'Agence de Bassin. En pratique, il est probable que dans l'un et l'autre cas il sera perçu des taxes identiques.

Le montant des taxes peut être calculé sur deux bases :

- Soit à partir de valeurs forfaitaires qui attribuent pour chaque type d'industrie, une pollution donnée ne dépendant que de la capacité de l'usine.

- Soit à partir de la mesure de la pollution réelle. Pour qu'un tel régime soit appliqué, il faut que l'industriel en fasse la demande. Les frais de mesures sont alors à sa charge.

Les valeurs forfaitaires de pollution sont identiques pour toute la France. Elles sont basées sur les insolubles et sur les matières oxydables qui sont égales à

$$
2 \mathrm{DBO} 5+\mathrm{DCO}
$$

Pour l'industrie laitière (laiterie, fromagerie ou beurrerie) on a les valeurs suivantes par $\mathrm{m}^{3}$ de lait travaillé :

- $1,20 \mathrm{~kg}$ d'insolubles

- 3,60 kg de matières oxydables.

Si on travaille à partir de crème, les valeurs de pollutions rapportées au $\mathrm{m}^{3}$ de crème sont à multiplier par 3 .

$\mathrm{Au}$ cas ou sérums et babeurres sont rejetés, il faudra ajouter aux flux de pollution déjà calculés, $28 \mathrm{~kg}$ de matières en suspension et $46 \mathrm{~kg}$ de matières oxydables par $\mathrm{m}^{3}$ de sérum ou de babeurre rejeté.

Le montant de la taxe est obtenu en multipliant les valeurs ci-dessus par le tonnage d'un jour d'activité normale du mois le plus chargé et en affectant le résultat d'un coefficient qui dépend 
de la localisation du point de rejet et qui peut être compris entre 0,5 et 2 .

On multiplie enfin le résultat par la valeur en $\mathrm{F}$ du $\mathrm{kg}$ d'insoluble ou du $\mathrm{kg}$ de matières oxydables. Ces taux de base sont variables suivant les Agences Financières de Bassins et oscillent entre $10,20 \mathrm{~F}$ et $21,70 \mathrm{~F}$ pour le $\mathrm{kg}$ d'insolubles et entre $10,20 \mathrm{~F}$ et $34,20 \mathrm{~F}$ pour le $\mathrm{kg}$ de matières oxydables.

Il est prévu des seuils au-dessous desquels la taxe n'est pas perçue et des réductions pour les premières années.

Il est évident que les taxes ci-dessus sont applicables s'il n'y a aucune épuration des eaux résiduaires. Elles peuvent être réduites, ou mieux, supprimées si l'industriel les traite avant rejet.

Les sommes dont disposeront désormais les Agences seront réutilisées par elles, après déduction des frais de fonctionnement, pour aider à la réalisation de travaux d'intérêt commun (études, réalisations de stations d'épuration, etc.). Les industriels sont donc en droit de demander l'aide des Agences pour les travaux qu'ils comptent entreprendre dans le domaine de l'eau.

\section{II. - POLLUANTS DES EAUX USEES DE LAITERIE}

On peut trouver dans les effluents de laiterie deux catégories principales de polluants :

- Les produits laitiers eux-mêmes, matières premières ou produits finis.

- Les réactifs utilisés pour les lavages et qui sont le plus souvent des produits acides (acide nitrique), des produits basiques (à base de soude) et des produits stérilisants (eau de javel). Il s'agit essentiellement de réactifs minéraux qui n'entraînent pas de pollution spécifique (les composés minéraux n'apparaissant pas dans les textes officiels), et qui sont de toute façon en faible concentration. Par contre, ils peuvent entraîner des pointes de $\mathrm{pH}$ soit dans le domaine basique, soit dans le domaine acide. Il faut également signaler le risque de pollution accidentelle qui peut exister du fait de la présence de compresseur à ammoniac. Il est souhaitable de prendre des précautions préliminaires pour éviter le rejet massif d'ammoniac en cas de rupture de tuyauteries.

Les produits laitiers se caractérisent, en ce qui concerne la pollution par une teneur en insolubles et par une DBO 5 élevée. En fait, il est surtout important de considérer la DBO car les matières en suspension sont difficiles à séparer et sont constituées surtout de matières organiques qui ont elles-mêmes une DBO. 
On trouve les valeurs suivantes pour quelques produits courants :

- Lait entier (à 3,6 p. 100 de mat. grasses) ..... .. . 103000 mg/l de DBO5

- Lait écrémé (à 0,1 p. 100 de mat. grasses) .. . . . $73000 \mathrm{mg} / 1$ de DBO5

- Lait concentré (à 7,9 p. 100 de mat, grasses) . ... . $208000 \mathrm{mg} / \mathrm{l}$ de DBO5

- Lait en poudre écrémé (à 0,9 p. 100 de mat. grasses) $737000 \mathrm{mg} / \mathrm{kg}$ de DBO5

- Crème (à 40 p. 100 de mat. grasses) .. . . . . . . . . . . $399000 \mathrm{mg} / \mathrm{l}$ de DBO5

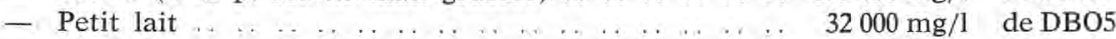

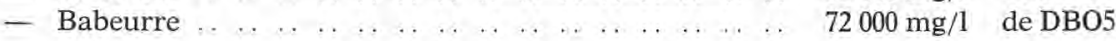

On voit que ces valeurs sont particulièrement élevées, mais il $\mathrm{DBO}$

est important de noter que pour le lait le rapport $\frac{}{\mathrm{DCO}}$ est relati-

vement proche de 1 , ce qui traduit la bonne aptitude à la dégradation biologique des composés chimiques contenu dans le lait. Cette remarque ne fait que confirmer que le lait constitue aussi pour les micro-organismes un excellent aliment équilibré en azote et phosphore et qu'il est possible d'épurer les effluents contenant du lait par un processus biologique.

Il faut cependant signaler que le babeurre et le sérum n'ont pas DBO DBO

les mêmes rapports $\frac{}{\mathrm{N}}$ et $\frac{\mathrm{P}}{\mathrm{P}}$ que le lait. Ils seront donc moins aptes à la synthèse de matière vivante, ce qui explique la difficulté de leur élimination quand ils ne sont pas mélangés avec des produits plus riches qu'eux en azote et phosphore.

Il est bien évident que le lait n'est pas un produit toxique en Iui-même. S'il est rejeté en quantité modérée dans les rivières il constituera un bon aliment pour les poissons. Si les masses évacuées dans le milieu naturel augmentent, la DBO du milieu va augmenter et deviendra à un moment supérieure à la capacité de réoxygénation. La teneur en oxygène dissous va donc diminuer et il en résultera un danger de mort par asphyxie du poisson, alors même qu'il se trouve en présence d'une nourriture abondante.

Ceci explique que l'on voit souvent de nombreux poissons autour d'une buse de rejets de laiterie, alors qu'en aval là ou l'oxygène dissous a disparu les poissons meurent.

II faut remarquer également que la pollution par des produits laitiers est provisoire, leur caractère de biodégradabilité qui fait leur danger, entraîne également qu'ils sont progressivement détruits et que la rivière retrouve, après un certain temps, une qualité sinon d'origine du moins suffisante.

Il apparaît ainsi que les risques présentés par les rejets ne seront pas proportionnels à l'augmentation de sa capacité (donc de ses rejets) mais pourront atteindre un seuil critique au-delà duquel la vie aquatique ne sera plus possible en aval du point de rejet. 


\section{III. - ORIGINES DE LA POLLUTION}

L'eau est utilisée à deux fins essentielles dans une usine laitière :

- Le refroidissement.

- Le lavage des appareils, des récipients et des sols.

Le refroidissement n'entraîne pas de pollution autre que thermique. Il est cependant assez fréquent de trouver des eaux dont la température dépasse parfois le seuil de $30^{\circ} \mathrm{C}$ fixé par la législation.

Les opérations de lavages sont celles qui sont à l'origine de la pollution ; elles entraînent, ainsi que nous l'avons vu, le rejet de lait ou de produits laitiers et des produits utilisés pour le lavage. $\mathrm{La}$ pollution essentielle sera due au lait et aux produits laitiers et sera ainsi due aux pertes qui peuvent exister à chaque poste.

Il est évident que pour une même perte de lait, les concentrations en DBO seront fonction de la quantité d'eau utilisée. Il faut noter que les concentrations moyennes sont de beaucoup supérieures aux normes et que pour les atteindre il faudrait une dilution tout à fait considérable. D'autre part, les taxes sont basées sur des flux de pollution et non plus des concentrations ; les volumes d'eau utilisés n'interviennent donc plus.

Nous allons essayer de passer en revue les différents stades par lesquels peut passer le lait et nous donnerons quelques valeurs des flux spécifiques de pollution qui peuvent provenir de chacun d'eux. Il s'agira bien entendu de valeurs moyennes qui ne seront qu'indicatives.

Il faut souligner une des caractéristiques importantes de la pollution laitière : elle provient d'opérations (lavages) qui sont essentiellement discontinues. On observera des concentrations qui varient beaucoup au cours du temps et qui rendent difficiles des mesures de pollution particulière.

\subsection{Réception du lait}

Le lait est reçu par l'usine, en bidons ou parfois en citernes. La pollution est due au lait perdu qui reste au fond des récipients avant leur lavage et dans une mesure beaucoup moindre, au lait répandu par terre au cours de son transfert. Plus les récipients qui sont utilisés sont petits, plus la perte du lait (et donc la pollution) est proportionnellement forte.

La perte de lait à ce poste est souvent évaluée à 2 à 3 p. 1000 de la quantité reçue.

Une source supplémentaire de pollution à ce poste est le lavage de la carrosserie des camions. Il entraine le rejet d'insolubles en quantité souvent importante, mais n'est pas la source de beaucoup de DBO. 


\subsection{Tanks de stockage}

La pollution, là encore est due au lait qui reste dans les récipients au moment où ceux-ci sont nettoyés. La forme des cuves, la place des tuyauteries de vidange auront une importance sur la quantité résiduelle du lait.

Plusieurs expériences ont montré que les pertes étaient souvent notablement plus importantes à ce poste qu'au lavage des bidons et des citernes de ramassage.

\subsection{Pasteurisateur}

La quantité de DBO5 rejetée au moment du lavage de pasteurisateurs peut être très variable suivant l'opérateur. Au cours de cette opération, on envoie en effet la solution de lavage par le circuit du lait. La pollution sera faible si la grande partie du lait contenu dans l'appareil est récupérée avant l'ouverture de la vanne de rejet. Elle peut être beaucoup plus intense si tout le lait est perdu (cette hypothèse est celle qui se rapproche le plus souvent de la réalité).

\subsection{Fromagerie}

Là encore la source essentielle de la pollution sera due aux opérations de lavage du matériel. Dans un atelier de ce type cependant la majeure partie des produits rejetés ne sera plus le lait, mais le sérum. Or, si le lait possède une valeur économique importante qui entraîne le souci d'en perdre le moins possible, le sérum est un sous-produit dont les industriels ont souvent du mal à se débarrasser. Il est donc commode d'en rejeter le maximum à l'égout.

La pollution d'un atelier de fromagerie sera donc essentiellement variable suivant le taux de récupération de sérum. Rappelons cependant que tant au point de vue redevances qu'au point de vue épuration, le rejet de sérum est à limiter au maximum.

Signalons que le lavage des toiles d'égouttage et le lavage du circuit éventuel des pâtes représentent de loin les opérations les plus polluantes dans une fromagerie.

\subsection{Beurrerie}

Le babeurre présente, en ce qui concerne la pollution, les mêmes inconvénients que le sérum. Il est de l'intérêt de l'industriel d'éviter au maximum son rejet avec les eaux usées.

L'eau de lavage du beurre est normalement évacuée. Il s'agit d'eaux usées qui sont, en général, très chargées et dont la DBO5 atteint plusieurs milliers de $\mathrm{mg} / \mathrm{l}$. Les débits sont en général assez faibles.

\subsection{Ateliers de poudre de lait}

Lorsque la poudre est fabriquée dans des tours, celles-ci ne sont lavées qu'à des intervalles de temps assez éloignés (de l'ordre d'une semaine). La quantité de DBO qui est alors rejetée peut être impor- 
tante car on a souvent affaire à des appareils de grande taille et parce que la poudre de lait possède une DBO très élevée.

La pollution peut dépendre de la qualité de la poudre fabriquée : elle semble être maximum lorsque l'on prépare des aliments bétail avec du lait écrémé réengraissé avec du suif. La poudre colle alors beaucoup plus aux parois et les pertes sont plus importantes.

Pour la fabrication de lait en poudre écrémé, les pertes sont de l'ordre de 4 p. 1000.

\subsection{Eau de lavage des sols}

Elle représente souvent un gros débit, qui peut être de l'ordre de la moitié de la consommation générale de l'usine (refroidissements exceptés), mais cette opération n'apporte pas une DBO importante. Dans beaucoup de cas ; il est possible d'envisager une réduction de cette consommation par des consignes d'exploitation, par l'installation de robinets appropriés et parfois par la réutilisation de certaines eaux (eau d'évaporation par exemple, s'il existe des installations de concentration).

\subsection{Effluents généraux}

Le mélange de tous les effluents particuliers d'une usine laitière présente un intérêt essentiel pour le calcul des installations d'épuration. Il présente les caractéristiques principales suivantes :

- Les pointes ont une amplitude plus faible que pour les effluents partiels. Ces pointes, tant en débit qu'en concentration, restent importantes et la DBO par exemple est susceptible de varier entre moins de 100 et plus de $1000 \mathrm{mg} / \mathrm{l}$.

- Les $\mathrm{pH}$ peuvent avoir des valeurs très variables comprises par exemple entre 6 et 12 .

- La mesure des matières en suspension est difficile du fait des possibilités de coagulation en fonction du $\mathrm{pH}$.

- La pollution varie au cours de la journée et au cours de l'année, en fonction de la production (celle-ci est en général maximum au printemps).

- Les eaux sont putrescibles et ne peuvent être stockées longtemps sans aération.

- Pour certaines usines, celles en particulier qui font du lait en poudre réengraissé, les effluents contiennent des matières grasses décantables.

A titre d'exemple, nous pouvons prendre le cas d'une laiterie recevant par jour :

- 1800001 de lait entier

- $100000 \mathrm{l}$ de lait écrémé

et fabriquant des pâtes fraîches et du lait en poudre écrémé (aliment bétail). 
Cette usine consomme pour ses lavages environ $500 \mathrm{~m}^{3} / \mathrm{jour}$ d'eau et rejette de l'ordre de $300 \mathrm{~kg} /$ jour de DBO, ce qui représente approximativement une perte de lait de 1 p. 100 de la production journalière. (Le sérum est entièrement récupéré). La concentration moyenne en DBO5 ressort ainsi à environ $600 \mathrm{mg} / 1$.

Cette usine utilise parallèlement $2000 \mathrm{~m}^{3} /$ jour d'eaux de refroidissement qui sont rejetées sans avoir été polluées chimiquement.

La répartition de la pollution entre les différents ateliers est la suivante :

- Laiterie (lavage des bidons, des tanks, pasteurisateur) environ 75 p. 100 de la DBO et 65 p. 100 du débit d'eaux de lavage (pour 1800001 par jour de lait entier).

- Atelier de pâtes fraîches : 4 p. 100 de la DBO et 10 p. 100 du débit d'eaux de lavage pour 24000 1/jour de lait passant dans cette unité (le sérum est entièrement récupéré).

- Fabrication de poudre de lait écrémé $(210000 \mathrm{l} / \mathrm{j})$, environ 22 p. 100 de la DBO et environ 25 p. 100 du débit d'eau de lavage.

On constate que les concentrations moyennes dans les différents ateliers sont du même ordre de grandeur et que la plus grande partie de la pollution vient de la première partie du circuit lait dans l'usine.

Dans ce cas, on note que l'on consomme pour les lavages d'appareils ou de récipients de stockage, environ $1 \mathrm{~m}^{3}$ d'eau par $\mathrm{m}^{3}$ de lait traité. Les autres consommations d'eau (sanitaires, lavage des sols...) doublent ce chiffre.

Ces valeurs n'ont qu'un caractère documentaire car on constate des différences importantes suivant les usines. Celles-ci sont dues à la nature des fabrications mais aussi à la nature du matériel, aux techniques utilisées pour les lavages et même au personnel d'exploitation. Il serait donc dangereux de vouloir extrapoler ces résultats pour n'importe quelle usine et il est de l'intérêt de l'industriel de vérifier avec soin la pollution qu'il apporte et les moyens de la réduire de l'intérieur même de l'usine.

Il apparaît cependant intéressant de noter que pour cette usine la pollution réelle est assez nettement inférieure à celle qui serait calculée à partir du barême de pollution établi pour la perception des redevances sur les rejets.

\section{IV. - PROCEDES D'EPURATION DES EAUX USEES}

\subsection{Procédés envisageables.}

Nous avons vu que la pollution des eaux de laiterie se caractérisaient essentiellement par leur $\mathrm{DBO}$ et que le rapport $\mathrm{DCO} / \mathrm{DBO}$ est relativement proche de 1 . Ceci signifie que les produits organi- 
ques polluants sont susceptibles d'être détruits par l'action des micro-organismes. Il est tout naturel alors d'utiliser cette aptitude pour l'épuration des eaux.

Il est bien entendu possible, sur le plan théorique, d'imaginer d'autres processus d'épuration. On constate en fait que les techniques de séparation de solides sont sans grands effets et que les procédés chimiques ou physico-chimiques d'élimination de produits dissous sont difficiles à mettre en œuvre et coûteux.

En pratique, seule l'épuration biologique est appliquée au traitement des eaux résiduaires de laiterie. Cette technique peut prendre deux aspects différents :

- L'épandage.

- Les boues activées.

\subsubsection{L'épandage}

L'épandage consiste à disperser sur le sol les eaux résiduaires. L'épuration se fait au cours de la traversée de la couche de terre. Pour qu'une telle opération soit efficace, il faut que les surfaces utilisées soient importantes et que la filtration soit assez lente. On observe malgré toutes les précautions un colmatage du terrain et l'impossibilité d'utiliser cette technique par temps humide alors que la terre est déjà gorgée d'eau.

L'épandage a pendant longtemps été utilisé de façon courante mais rarement dans des conditions satisfaisantes. Le développement de la taille des usines fait qu'il est de plus en plus difficile à appliquer et les Pouvoirs Publics le déconseillent assez fortement.

Il faut remarquer, en outre, que l'épandage demande un investissement qui est loin d'être négligeable. Il faut prévoir des tuyauteries qui peuvent être longues et de la main-d'œuvre pour déplacer ces installations.

L'épandage ne peut plus, à notre avis, être considéré comme une technique d'épuration applicable aux unités modernes.

\subsubsection{Les boues activées}

L'épuration par boues activées consiste à reproduire artificiellement les conditions qui existent dans une rivière et qui assurent son autoépuration. Ceci consiste à maintenir en présence des microorganismes, de l'oxygène (c'est-à-dire de l'air) et l'eau à traiter. Il est bien évident que l'on opère avec des quantités considérables de micro-organismes de façon à avoir des vitesses de réaction très supérieures à celles qui sont observées dans le milieu naturel qui est dilué.

En pratique on réalise des bassins dans lesquels sont envoyés les eaux usées à traiter. L'air est introduit par un moyen mécanique quelconque, turbines de surface, bullage, brosses, etc. Les microorganismes sont introduits au démarrage et se développent naturel- 
lement du fait de la présence d'un milieu nutritif favorable. On maintient leur concentration dans ce bassin au moyen d'un recyclage à partir du décanteur secondaire.

\section{Figure 1}

Station d'épuration de l'Ucalma

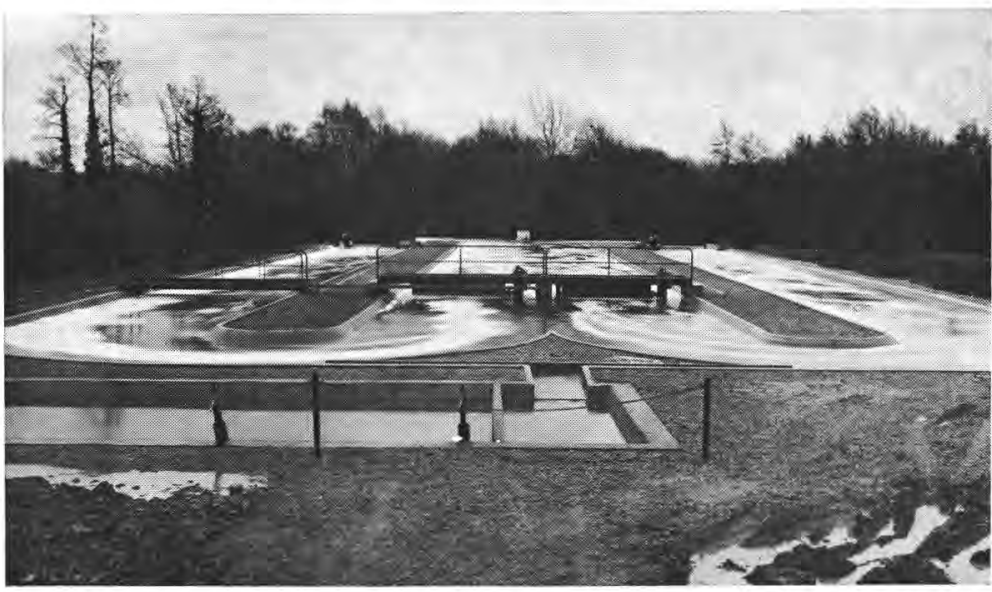

(Gaudiat photographe, 56, rue de Douai, Paris $9 e$

En effet, les micro-organismes responsables de l'épuration sont en suspension dans le milieu. Il faut donc les séparer de l'eau pour pouvoir rejeter celle-ci. On place donc à la suite du bassin d'aération un appareil séparateur, qui est presque toujours un décanteur, d'où l'eau débarrassée des insolubles est rejetée. Les solides séparés, qui contiennent les micro-organismes sont en majeure partie recyclés dans le bassin. Une autre partie, qui représente l'excès de boues qui s'est formé au cours de l'épuration est évacuée.

Les matières organiques détruites au cours de l'épuration servent, pour une part, à former de la matière vivante. Il y a donc transfert des polluants avec disparition de la DBO et apparition de solides.

Ces solides dans un premier stade sont putrescibles, ce qui rend difficile leur évacuation, car celle-ci doit être très rapide. Dans la très grande majorité des cas, on se trouve donc contraint de stabiliser ces solides pour éviter leur évolution ultérieure.

Pour traiter ces boues on utilise presque toujours un processus biologique qui revient à l'autodestruction des micro-organismes. Ce processus peut être aérobie ou anaérobie. 
La stabilisation a pour conséquence de réduire la masse de solides à extraire. Elle présente donc le double avantage de diminuer les tonnages et de faciliter leur manipulation ou leur stockage.

Le traitement anaérobie ne nécessite bien entendu pas l'introduction d'oxygène, ce qui oblige de dépenser de l'énergie. Il se fait dans des appareils fermés qui sont coûteux d'investissement. Il y a production de gaz combustibles qui sont utilisés pour réchauffer la masse (donc augmenter la vitesse et réduire la taille des appareils). La digestion anaérobie est employée de façon courante pour l'épuration des eaux usées urbaines pour lesquelles on utilise aussi un procédé biologique. Pour les laiteries son intérêt est beaucoup plus réduit. En effet, il est très important d'éviter l'apparition de fermentations acides au cours de la digestion. Ce type de fermentation est une évolution normale pour des eaux contenant des produits laitiers. Il existe alors un risque très important de mauvais fonctionnement d'un digesteur et cette technique est peu utilisée.

Le traitement aérobie des boues découle de l'observation qui a été faite, montrant qu'il existe trois phases au cours d'un processus biologique :

- La première phase est l'adsorption par les bactéries, des produits nutritifs qui sont responsables de la DBO. Il s'agit d'un phénomène, en grande partie, physique et qui est rapide. L'eau se trouve épurée mais les polluants ne sont pas encore profondément transformés. Il n'y a eu que transfert de la pollution.

- Une seconde phase est l'assimilation par les micro-organismes, de la nourriture adsorbée. Une partie est oxydée, l'autre sert à la production de nouvelle matière vivante. Il y a transformation de la pollution et consommation d'oxygène. Cette phase est plus lente mais est rendue possible du fait que le temps de séjour des boues dans le bassin est, à cause du recyclage, plus élevé que le temps de séjour de l'eau.

- La dernière phase est l'autodestruction des micro-organismes qui est un processus lent et dont l'importance relative n'est grande que dans un milieu pauvre en DBO. C'est ce que l'on appelle la respiration endogène.

Dans un bassin à boues activées les trois phases se font simultanément, des bactéries existant à chacune de ces étapes. Pour mettre à profit l'autodestruction des micro-organismes, deux techniques existent :

- Soit maintenir dans le bassin pendant un temps prolongé les micro-organismes. Pour arriver à ce but, il est évident qu'il faut des bassins de grande taille où la quantité de DBO par unité de temps et par unité de volume est faible. On a alors ce qui est appelé en général l'oxydation totale.

- Soit extraire du bassin de traitement des boues non encore stabilisées et les envoyer dans un autre bassin où elles sont aérées 
pendant un temps prolongé. Cette technique présente comme principal avantage de conduire à un volume total d'aération nettement plus faible que dans le cas précédent. Elle conduit par contre à des installations plus compliquées qui ne sont économiques en investissement qu'à partir d'une certaine taille.

Dans les deux cas les consommations d'énergie sont comparables : elles atteignent au maximum $1 \mathrm{kwh}$ par $\mathrm{kg}$ de DBO éliminée.

Une dernière étape éventuelle est la déshydratation des boues résiduelles qui existent toujours. Il faut en effet être conscient de ce que les solides à évacuer sont toujours extrêmement dilués et qu'ils représentent donc des volumes assez considérables. La déshydratation peut se faire sur des lits de séchage qui sont des filtres de très grande dimension à ciel ouvert, où l'évaporation joue son rôle. Les lits de séchage sont malheureusement très sensibles aux conditions atmosphériques et leur fonctionnement est bien souvent décevant. Il existe aussi des procédés mécaniques, filtration sous vide, centrifugation, coûteux et demandant de la main-d'œuvre. Ils sont de ce fait difficilement utilisables dans les usines qui ne sont pas de très grande taille.

Nous devons signaler pour mémoire que l'épuration biologique peut en principe se faire dans un autre type d'appareil que sont les lits bactériens (appelés encore filtres biologiques). Dans ce cas les micro-organismes sont fixés sur un support solide à large maille (cailloux, ponzzolane), sur lequel ont fait ruisseler l'eau à traiter. L'air circule dans le milieu, soit naturellement du fait de la différence de température, soit à l'aide d'un ventilateur.

Les lits bactériens consomment peu d'énergie mais ils sont de moins en moins employés en laiterie. Le risque le plus important est le colmatage par les graisses, colmatage qui rend nécessaire de refaire le lit.

Enfin pour certaines petites installations, la décantation se fait dans le bassin d'aération lui-même qui a alors un fonctionnement pulsé : alimentation avec l'eau et aération, arrêt de l'agitation et de l'alimentation, rejet de l'eau surnageante. Malgré les économies auxquelles conduit une telle technique, elle est d'exploitation plus délicate et ne peut convenir en pratique que pour les petites installations.

\subsection{Recommandations}

Nous venons de voir les principes des techniques applicables à l'épuration des eaux usées de laiterie. Leur application est cependant loin d'être d'une grande simplicité et en chaque circonstance il faut tenir compte des situations locales. Nous allons essayer d'envisager ci-dessous les précautions à prendre au moment oì il sera envisagé la construction d'une station d'épuration. 


\subsubsection{Définition des charges polluantes et des débits}

Cette définition demande un soin tout particulier car l'installateur consulté calculera ses appareils à partir de ces renseignements. Sous évaluer ces grandeurs conduira presque certainement à une station marchant mal et qui sera la source de plus de difficultés qu'une absence d'épuration.

En pratique il n'existe qu'un seul moyen sûr : la réalisation de mesures sur un temps suffisamment long pour être représentatif. Les résultats obtenus devront être éventuellement corrigés pour tenir compte des périodes de pointe et des perspectives d'extension à court ou moyen terme. Il pourra d'ailleurs apparaître au cours d'une telle étude préliminaire, la possibilité de réduire par des moyens simples, la consommation d'eau et la pollution rejetée ; de telles constatations suffisent souvent, à elles seules, à compenser le coût de l'étude, par les réductions de dépenses qu'elles entraînent.

\subsubsection{Prévisions des risques de pollution accidentelle}

Il est important de connaître et si possible d'éliminer les risques de rejets accidentels d'ammoniac par exemple ou de grandes quantités de lait ou de produits de nettoyage. Il faudra également savoir si la possibilité existe d'avoir à envoyer avec les eaux de grandes quantités de sérum ou de babeurre à certaines époques.

\subsubsection{Bassin tampon}

Nous avons vu que la pollution et les rejets étaient variables au cours du temps, la plupart des opérations consommatrices d'eau étant discontinues dans une laiterie.

Afin de diminuer la taille des installations à mettre en œuvre et pour leur donner un fonctionnement aussi stable que possible, il est tout à fait souhaitable de répartir les rejets sur le maximum d'heures dans la journée. Il apparaît alors le très grand avantage de construire des bassins tampons dont les dimensions seront fonction de la courbe des débits et des pollutions au cours de la journée. Il faut cependant faire attention aux risques de fermentation. Si donc le temps de séjour dans le bac tampon doit dépasser quelques heures, il sera nécessaire de l'aérer (ce que constituera d'ailleurs une pré-épuration).

Un tel bac tampon peut servir d'ailleurs à la séparation des graisses décantables. Il permet en outre une régularisation des $\mathrm{pH}$, les eaux de laiterie étant en moyenne, voisines de la neutralité.

\subsubsection{Sérum et babeurre}

En principe, on doit éviter leur rejet, mais il est parfaitement admissible qu'une partie de la DBO ait ces sous produits comme origine. Il est alors important de connaître les quantités évacuées. 
A la limite, si celles-ci sont trop élevées, il pourra apparaître la nécessité d'ajouter à l'effluent azote et phosphore destinés à équilibrer la composition de l'effluent.

\subsubsection{Boues}

Presque toutes les installations d'épuration d'effluents de laiterie donnent naissance à des boues résiduelles qui doivent être évacuées. Ces boues sont des suspensions diluées de solides non putrescibles pour une station bien conçue.

Avant la mise en route il faut savoir quelle pourra être l'utilisation de ces résidus. Ils peuvent être employés comme engrais mais l'expérience prouve qu'on a souvent du mal à s'en débarrasser même gratuitement.

Une autre solution est l'épandage, solution qui est admissible en raison du volume relatif faible par rapport au volume des eaux.

Enfin si aucune évacuation des boues diluées n'est possible, il faudra envisager leur concentration et éventuellement leur déshydratation.

\subsubsection{Circuits d'eau}

Beaucoup d'usines laitières ont des circuits séparatifs. Ce système est très souvent avantageux car il évite d'avoir à envoyer à la station des eaux non polluées (eaux de refroidissement ou eaux de pluie).

Cependant si la concentration des effluents à traiter dépasse 2000 à $3000 \mathrm{mg} / 1$ de $\mathrm{DBO}$, il peut être intéressant de les diluer afin de faciliter l'épuration. Ce cas reste exceptionnel, et il est encore plus rare qu'il conduise à recommander un réseau unitaire intégral.

\section{Conclusions}

Les eaux usées de laiterie posent un problème d'épuration de plus en plus important, notamment en raison de l'accroissement de la taille des usines. Ainsi, celle que nous avons citée dans cet article rejette une quantité de DBO qui équivaut à celle d'une ville de 6000 habitants environ (dans un volume d'eau beaucoup plus réduit).

Il est cependant possible d'épurer de façon satisfaisante de telles eaux usées et les installations bien conçues n'ont pas des coûts d'exploitation très élevés et ne demandent qu'une surveillance restreinte facilement assurée par le personnel de l'usine.

Il s'agit néanmoins d'un problème délicat qui nécessite des études préalables sérieuses qui seules permettent de définir avec précision le problème à résoudre et les solutions à lui appliquer. Il s'agit là d'un travail de spécialistes. Il faut en effet être conscient que chaque usine a des aspects du problème qui lui sont propres 
et qu'une station d'épuration demande d'être étroitement adaptée en fonction des eaux à traiter et des performances qui lui sont demandées.

\section{Conclusions}

Dairy wastes raise more and more acutely the problem of waste water treatment, because of the increasing capacities of dairy-plants. In the above-mentionned instance, the quantity of rejected DBO is that of a town of about 6000 inhabitants (in a much smaller quantity of water).

Yet it is possible to treat such water in a satisfactory way ; in well thought-out fittings working expenses are not very high, and control can be easily given to the staff.

Nevertheless the problem is difficult and demands serious preliminary studies, which alone can determine the problem that is to be solved, and the solutions that are to be used. It can only be the work of specialists. It is necessary to remember that each dairy plant presents its own aspects of the problem, and that a waste water treatment plant has to be adapted to the waters it will treat, and to the efficiency which is demanded.

Reçu pour publication en mars 1969. 